\title{
Effective Transcutaneous Delivery of Hyaluronic Acid Using an Easy-to-Prepare Reverse Micelle Formulation
}

\author{
Shuto Kozaka ${ }^{1}$, Ayaka Kashima ${ }^{1}$, Rie Wakabayashi ${ }^{1,2}{ }^{\circledR}$, Takahiro Nakata ${ }^{3}$, Taro Ueda ${ }^{3}$ and \\ Masahiro Goto $1,2,4, *$ (D) \\ 1 Department of Applied Chemistry, Graduate School of Engineering, Kyushu University, Motooka 744, \\ Nishi-ku, Fukuoka 819-0395, Japan; kozaka.shuto.119@s.kyushu-u.ac.jp (S.K.); \\ cheitseiutvn52g8djgwanvdms@gmail.com (A.K.); rie_wakaba@mail.cstm.kyushu-u.ac.jp (R.W.) \\ 2 Center for Future Chemistry, Kyushu University, Motooka 744, Nishi-ku, Fukuoka 819-0395, Japan \\ 3 Kobayashi Pharmaceutical Co., Ltd. 1-30-3 Toyokawa, Ibaraki, Osaka 567-0057, Japan; \\ ta.nakata@kobayashi.co.jp (T.N.); t.ueda@kobayashi.co.jp (T.U.) \\ 4 Advanced Transdermal Drug Delivery System Center, Kyushu University, Motooka 744, Nishi-ku, \\ Fukuoka 819-0395, Japan \\ * Correspondence: m-goto@mail.cstm.kyushu-u.ac.jp; Tel.: +81-92-802-2806
}

Received: 21 May 2020; Accepted: 28 June 2020; Published: 1 July 2020

\begin{abstract}
The skin loses its moisture with advancing age, causing cosmetic issues such as wrinkles. In addition, the loss of moisture leads to hypersensitivity to external stimuli such as UV light. Transcutaneous supplementation with hyaluronic acid (HA) is an effective and safe method of recovering the moisturizing function and elasticity of the skin. However, the transcutaneous delivery of HA remains challenging owing to the barrier function of the stratum corneum (SC) layer. To penetrate the SC barrier, we used a reverse micelle formulation that does not require high energy consumption processes for preparation. We aimed to enhance the skin permeability of HA by incorporating glyceryl monooleate-a skin permeation enhancer-into the formulation. A fluorescently-labeled HA-loaded reverse micelle formulation showed significantly enhanced permeation across Yucatan micro pig skin. Fourier transform infra-red spectroscopy of the surface of the skin treated with the reverse micelle formulation showed blue shifts of the $\mathrm{CH}_{2}$ symmetric/asymmetric stretching peaks, indicating a reduction in the barrier function of the SC. Further study revealed that HA was released from the reverse micelles at the hydrophobic/hydrophilic interface between the SC and the living epidermis. The results demonstrated that our reverse micellar system is an easy-to-prepare formulation for the effective transcutaneous delivery of HA.
\end{abstract}

Keywords: cosmetics; biomedicine; transcutaneous drug delivery; hyaluronic acid; reverse micelle

\section{Introduction}

The skin is the largest organ and plays an important role as a barrier, maintaining the homeostasis of the human body. However, with advancing age, the skin gradually loses its barrier function and moisture, causing cosmetic problems such as wrinkles. The dominant causes of these problems are internal factors, such as a loss of the extracellular matrix (ECM), and external factors such as exposure to UV light [1]. Skin with higher transepidermal water loss causes inflammation under lower doses of UV light [2]. Therefore, once the skin loses moisture, it enters a compounding cycle in which its greater sensitivity to external stimuli leads to further moisture loss. A key molecule in breaking this cycle is hyaluronic acid (HA) - a linear polysaccharide with repeating units of disaccharides of $\mathrm{N}$-acetyl-D-glucosamine and D-glucuronic acid-which is one of the components of the ECM and 
is mostly found in the human dermis [3]. HA is used in various biomedical fields, owing to its excellent biodegradability, biocompatibility, non-toxicity and non-immunogenicity [4-6]. Skin aging is associated with the loss of HA in the dermis; therefore, supplementation with HA is required to maintain the moisturizing function and elasticity of the skin. Injection has been used as an HA supplementation method; however, medical accidents, including the loss of eyesight following an HA injection to the face, have been reported $[7,8]$. Another method is oral administration; however, when applied this way, HA loses its function as a result of metabolism in the digestive organs. In contrast to these methods, transcutaneous delivery is a safe and effective administration method for HA because it does not require needles and can directly target the dermis without undergoing metabolism. However, the skin permeation of HA is prohibited by the topmost layer of skin, the stratum corneum (SC) layer [9]. The SC is composed of corneocytes and intercellular lipids that work as a barrier to molecules that are both large and hydrophilic, including HA. Although it has been reported that HA enhances the skin permeability of accompanying drugs by hydrating the SC [10-12], delivering HA itself into the skin remains a challenge. Physical enhancement techniques, such as microneedles [13] and an ultra-sound assisted system [14], have been developed; however, similarly to injection, safety concerns remain. Tokudome et al. successfully delivered HA into the dermis using polyion complex emulsions, without using physical enhancement techniques [15], and we reported successful HA delivery using the solid-in-oil technique [16]. However, these techniques require high cost processes such as freeze-drying and high-speed homogenizing, making them unfavorable for industrial application.

To overcome these limitations, we propose the use of reverse micelles that can encapsulate hydrophilic drugs in their aqueous core. The reverse micelle formulation is an easy-to-prepare system that is produced by mixing water, surfactant and oil components without high energy consumption processes. Numerous researchers have developed reverse micelle formulations with various surfactant molecules—such as phospholipids [17], fatty acids [18] and an amphiphilic dendritic polymer [19]—-to deliver small molecule drugs. However, there are only a few reports of the delivery of high molecular weight drugs ( $>500 \mathrm{Da}$ ) [20,21] and, to the best of our knowledge, no one has achieved the transcutaneous delivery of HA into the dermis layer using a reverse micelle formulation.

We previously developed a reverse micelle formulation, using glyceryl monooleate (MO) as the surfactant, and succeeded in delivering a peptide antigen ( 1400 Da) to the skin dendritic cells in the living epidermis layer [22]. The formulation was able to enhance the permeability of skin to such a high molecular weight drug owing to the strong permeation enhancing effect of MO. However, this system has not been applied for polysaccharides, including HA, and its permeation mechanism has not been fully addressed. Here, to expand the range of cargo molecules that can be delivered by our system, we first examined the optimal composition for encapsulating HA in the reverse micelle formulation. Subsequently, we evaluated the skin permeation of the HA-loaded reverse micelle formulation and examined the permeation mechanism using fluorescence imaging and Fourier transform infra-red (FTIR) spectroscopy. The results showed our reverse micelle system to be an easy-to-prepare formulation for the effective transcutaneous delivery of HA.

\section{Materials and Methods}

\subsection{Materials}

Hyaluronic acid (HA; MW = $10 \mathrm{kDa}$ ) was purchased from Kewpie (Tokyo, Japan). Isopropyl myristate (IPM) and 1-ethyl-3-(3-dimethylaminopropyl)-carbodiimide (EDC) were bought from Tokyo Chemical Industry (Tokyo, Japan). Isopropanol (IPA) was obtained from Kishida Chemical (Osaka, Japan). Glyceryl monooleate (MO) was purchased from Nikko Chemicals (Tokyo, Japan). Fluorescein-cadaverine dihydrochloride was obtained from Toronto Research Chemicals (North York, $\mathrm{ON}$, Canada), and 1-hydroxybenzotriazole monohydrate $\left(\mathrm{HOBt} \cdot \mathrm{H}_{2} \mathrm{O}\right)$ was bought from Watanabe Chemical (Osaka, Japan). Rhodamine labeled 1,2-dioleoyl-sn-glycero-3-phosphoethanolamine 
(Rh-DOPE) was purchased from Avanti Polar Lipids (Alabaster, AL, USA). Yucatan micro pig (YMP) skin was obtained from Charles River Laboratories Japan (Kanagawa, Japan).

\subsection{Synthesis of Fluorescein-Labeled HA (FCHA)}

Fluorescein cadaverine and HA were chemically conjugated using the EDC/HOBt coupling reaction. EDC in purified water $(48.5 \mathrm{mg} / \mathrm{mL})$ and $\mathrm{HOBt} \cdot \mathrm{H}_{2} \mathrm{O}$ in DMSO $(34.5 \mathrm{mg} / \mathrm{mL})$ were mixed at a 1:1 volume ratio (coupling reagent). Then, $1 \mathrm{~mL}$ of fluorescein-cadaverine dihydrochloride in DMSO $(7.56 \mathrm{mg} / \mathrm{mL})$ and $4 \mathrm{~mL}$ of HA in purified water $(12.5 \mathrm{mg} / \mathrm{mL})$ were mixed, and $2 \mathrm{~mL}$ of the coupling reagent was added to initiate the coupling reaction. The solution was stirred for $24 \mathrm{~h}$ at room temperature using a magnetic bar. Subsequently, the solution was transferred into a $3 \mathrm{kDa}$ cut-off dialysis membrane to remove residual fluorescein-cadaverine dihydrochloride. Finally, the solution was freeze-dried to obtain the FCHA powder.

\subsection{Preparation of the FCHA-Loaded Reverse Micelle Formulation}

Ahead of the preparation of the FCHA-loaded reverse micelle formulation, IPM and MO were heated to $70^{\circ} \mathrm{C}$ on a block incubator (EYELA, Tokyo, Japan). Precise amounts of MO and IPA were added to the IPM, and the mixture was stirred for several minutes with heating. Subsequently, the aqueous solution of FCHA was added dropwise. Finally, the mixture was vortexed and allowed to cool to room temperature. The final concentration of FCHA was $1.0 \mathrm{mg} / \mathrm{mL}$. Detailed compositions are stated in Table 1. The water content and the particle size of the FCHA-loaded reverse micelle formulation were determined by Karl Fischer titration and dynamic light scattering (Zetasizer Nano ZS, Malvern, Worcestershire, UK), respectively. For morphological observation, the FCHA-loaded reverse micelle formulation was sandwiched between a glass slide and a cover glass and observed with a confocal laser scanning microscope (CLSM, LSM700, Carl Zeiss, Jena, Germany). To evaluate the homogeneity of the reverse micelles, the dispersed fraction of the aqueous phase was defined as follows:

$$
\text { (Dispersed fraction of aqueous phase) }[\%]=R_{\text {water }}[\%] \cdot W_{R M}[\mathrm{~g}] / W_{\text {water }}[\mathrm{g}]
$$

where $R_{\text {water }}$ is the water content in the reverse micelles, $W_{R M}$ is the total weight of the reverse micelle formulation, and $W_{\text {water }}$ is the weight of water added to the formulation.

\subsection{In Vitro Skin Permeation Study}

YMP skin stored at $-80^{\circ} \mathrm{C}$ was defrosted at room temperature, and the fat was carefully removed in order to avoid damaging the dermis layer. Next, $1.5 \times 1.5 \mathrm{~cm}^{2}$ segments of the full-thickness YMP skin were fixed on Franz-type diffusion cells (effective diffusion area: $0.785 \mathrm{~cm}^{2}$ ), and the receiver phase was $5 \mathrm{~mL}$ of phosphate-buffered saline (PBS). All of the diffusion cells were equilibrated at $32.5^{\circ} \mathrm{C}$ to reproduce the temperature of the human skin surface. Then, $250 \mu \mathrm{L}$ of the FCHA-loaded reverse micelle formulation was added to the donor chamber and the whole experiment system was maintained at $32.5^{\circ} \mathrm{C}$ for $24 \mathrm{~h}$. The same amount of FCHA in PBS $(1.0 \mathrm{mg} / \mathrm{mL})$ was used as a control aqueous solution group. The skin segments were then washed with $20 \%$ ethanol prior to the imaging and quantification experiments. For the imaging experiment, the skin segments were soaked in 4\% paraformaldehyde (Fujifilm Wako Pure Chemical, Osaka, Japan) for $6 \mathrm{~h}$, frozen in liquid nitrogen, and embedded in an optimal cutting temperature compound. Subsequently, the skin segments were sliced into $20 \mu \mathrm{m}$ thicknesses with a microtome cryostat (Leica CM1860UV, Leica Biosystems, Wetzlar, Germany) and then adsorbed on glass slides. Specimens were covered with Entellan (Merck, Darmstadt, Germany) and a cover glass for fluorescence imaging with CLSM. For the quantification of FCHA, the skin segments were cut into small pieces with a scalpel and submerged in $500 \mu \mathrm{L}$ of PBS:methanol:acetonitrile $=2: 1: 1(\mathrm{v} / \mathrm{v} / \mathrm{v})$ to extract FCHA from the YMP skin. The extract solution was then filtered through a polytetrafluoroethylene membrane with $0.2 \mu \mathrm{m}$ pores, and the 
amount of FCHA that permeated into the YMP skin was quantified using the fluorescence intensity at $\lambda_{\mathrm{ex}}=493 \mathrm{~nm}$ and $\lambda_{\mathrm{em}}=521 \mathrm{~nm}$ (LS55, PerkinElmer, Waltham, MA, USA).

Table 1. Compositions of the fluorescein-labeled hyaluronic acid (FCHA)-loaded reverse micelle formulations $((\mathrm{FCHA})=1 \mathrm{mg} / \mathrm{mL}) . W_{0}$ is the water-to-MO (glyceryl monooleate) molar ratio.

\begin{tabular}{ccccc}
\hline IPM & IPA & MO & Water & $W_{\mathbf{0}}$ \\
\hline (wt. $\%)$ & (wt.\%) & (wt. $\%)$ & (wt. $\%)$ & $(-)$ \\
\hline 91.5 & 2.0 & & & \\
89.5 & 4.0 & & & \\
87.5 & 6.0 & 6.0 & 0.5 & 1.7 \\
85.5 & 8.0 & & & \\
83.5 & 10.0 & & & \\
\hline 89.2 & 2.0 & & & \\
87.2 & 4.0 & & & \\
85.2 & 6.0 & 8.0 & 0.8 & 2.0 \\
83.2 & 8.0 & & & \\
81.2 & 10.0 & & & \\
\hline 93.5 & 2.0 & & & \\
91.5 & 4.0 & & & \\
89.5 & 6.0 & 4.0 & 0.5 & 2.5 \\
87.5 & 8.0 & & & \\
85.5 & 10.0 & & & \\
\hline 91.0 & 2.0 & & & \\
89.0 & 4.0 & & & \\
87.0 & 6.0 & 6.0 & 1.0 & 3.3 \\
85.0 & 8.0 & & & \\
83.0 & 10.0 & & & \\
\hline
\end{tabular}

\subsection{In Vitro Skin Permeation Study}

Mouse ear auricles were used to elucidate how FCHA permeated through the SC layer. Defrosted ear auricles were treated with $10 \mu \mathrm{L}$ of the FCHA-loaded reverse micelle formulation for $2 \mathrm{~h}$ under moisture control. The ear auricles were then washed with PBS:methanol:acetonitrile $=2: 1: 1(v / v / v)$ and sandwiched between a glass slide and a cover glass for CLSM observation.

To elucidate the permeation mechanism, the nanostructure in the SC layer was analyzed by Fourier transform infra-red (FTIR) spectroscopy. First, an SC sheet was obtained, as previously reported $[23,24]$. Briefly, defrosted YMP skin was heated at $60^{\circ} \mathrm{C}$ for $90 \mathrm{~s}$ in a water bath, and the epidermis sheet was carefully peeled off with forceps. The epidermis sheet was then incubated in $5 \mathrm{~mL}$ of $0.25 \%$ trypsin- 1 mM ethylenediaminetetraacetic acid solution (Thermo Fisher Scientific, Waltham, MA, USA) overnight, at room temperature. The obtained SC sheet was washed with water and dried for further use. A piece of the SC sheet was treated with $100 \mu \mathrm{L}$ of the FCHA-loaded reverse micelle formulation at $32.5^{\circ} \mathrm{C}$ for $24 \mathrm{~h}$ and then washed and dried thoroughly. Finally, the FTIR spectrum of the SC sheet was obtained using a Spectrum Two (PerkinElmer) with a resolution of $1 \mathrm{~cm}^{-1}$ and 128 scans.

To observe the distribution of surfactants in the skin, Rh-DOPE was used as a fluorescent surrogate for MO. MO was combined with $0.015 \mathrm{wt} \% \mathrm{Rh}-\mathrm{DOPE}$ and used for the preparation of a dual-labeled reverse micelle formulation. The cross-section of YMP skin that was treated with dual-labeled reverse micelles was observed with CLSM using the same process as in 2.4.

\subsection{Statistical Analysis}

Statistical analysis was performed using Microsoft Excel 2019 (Microsoft, Redmond, DC, USA). The indicated $P$-values were derived from Student's t-test. Significance is indicated as ${ }^{*} P<0.05$, 
${ }^{* *} P<0.01,{ }^{* * *} P<0.001$ and ${ }^{* * *} P<0.0001$. Data are expressed as the mean \pm standard error of the mean value, unless specified.

\section{Results \& Discussion}

\subsection{Preparation and Characterization of the Reverse Micelle Formulation}

The chosen components of the reverse micelle formulation were IPM as the oil phase, MO as the surfactant, IPA as the co-surfactant and Milli-Q water as the aqueous phase. MO is an ester of glycerol and oleic acid and works as a strong skin permeation enhancer $[25,26]$. All of the components were non-toxic and pharmaceutically acceptable. In addition, the reverse micelle formulation could be produced by simply mixing all of the components, without the need for high energy consumption processes, which is favorable for industrial application. First, reverse micelles with the compositions outlined in Table 1 were prepared in order to determine the optimal formulation. As an indicator of the homogeneity of the reverse micelles, we defined the dispersed fraction of the aqueous phase, which means the percentage of added water dispersed into the formulation. If the dispersed fraction is $100 \%$, a formulation is completely homogeneous, unless the formulation causes phase separation or precipitation. The dispersed fraction had a positive correlation with the mass fraction of IPA (Figure 1). A likely reason for this observation is the surface activity of IPA, which can work as a co-surfactant in addition to MO, allowing a greater amount of water to be dispersed in the IPM. Another factor that governs the dispersed fraction is the molar ratio of water-to-MO $\left(W_{0}\right)$. As $W_{0}$ increased from 1.7 to 3.3 , the dispersed fraction tended to decrease. This tendency is consistent with the fact that more surfactant molecules, $\mathrm{MO}$ in this case, are required to reduce the surface free energy at the polar/nonpolar interface as the volume of water increases. Based on these results, we determined the optimal reverse micelle formulation to be $10 \mathrm{wt} \%$ IPA mass fraction and a $W_{0}$ value of 1.7 , with the composition IPM:IPA:MO:water = 83.5:10.0:6.0:0.50 (w/w/w/w), for which the dispersed fraction was nearly $100 \%$.

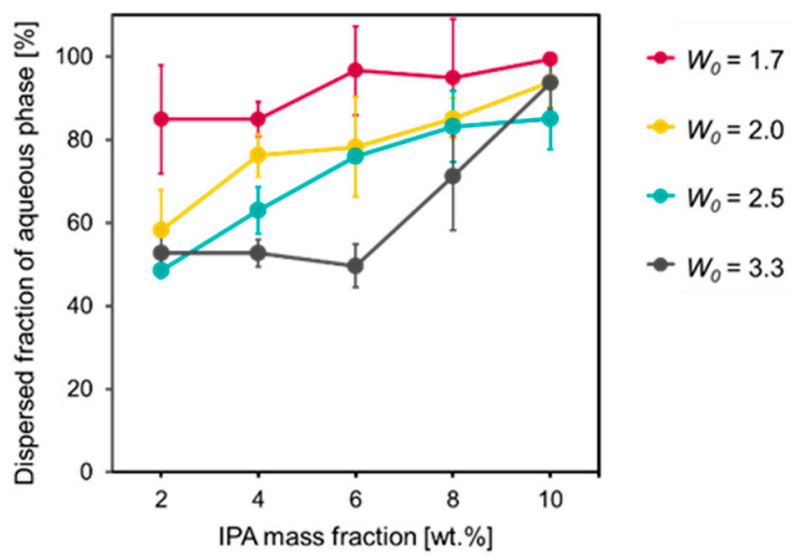

Figure 1. Dispersed fractions of aqueous phase in reverse micelle formulations versus various compositions $(n=3$, mean $\pm S E)$.

The FCHA-loaded reverse micelle formulation with the optimal composition was used for further evaluation. Dynamic light scattering measurement showed that the size distribution of the reverse micelles was monomodal and the Z-average was $871.8 \mathrm{~nm}$ (Figure 2a). Next, the FCHA-loaded reverse micelle formulation was observed with CLSM to directly evaluate the location of FCHA in the formulation. The fine polka-dot pattern of green fluorescence in the acquired fluorescence image indicates that FCHA was encapsulated in the hydrophilic domain of the reverse micelles (Figure 2b). These results show that the water phase containing the hydrophilic FCHA was uniformly dispersed in the organic phase (IPM). 


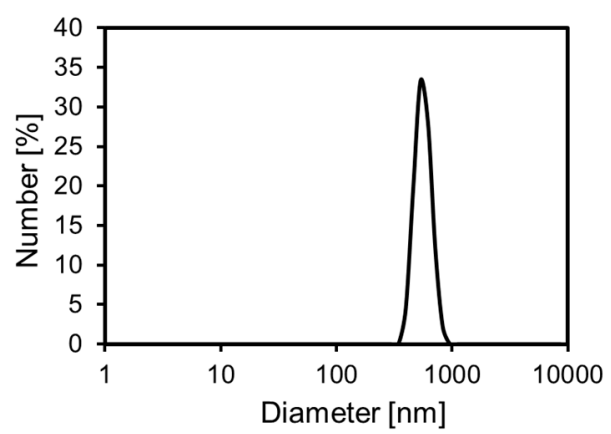

(a)

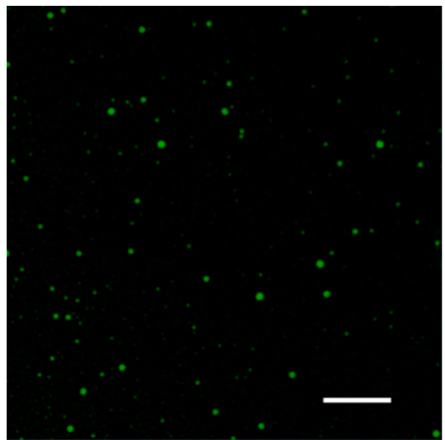

(b)

Figure 2. Characterization of the FCHA-loaded reverse micelle formulation. (a) Particle size distribution. (b) Confocal laser scanning microscope (CLSM) image with a $100 \times$ lens. Scale bar: $10 \mu \mathrm{m}$.

\subsection{Permeation of FCHA into YMP Skin}

YMP skin - a preferred in vitro animal skin model — was chosen to evaluate the skin permeation of the reverse micelle formulation [27]. First, cross-sections of YMP skin treated with FCHA in PBS or the FCHA-loaded reverse micelle formulation $((\mathrm{FCHA})=1.0 \mathrm{mg} / \mathrm{mL})$ were observed with CLSM to reveal the distribution of FCHA (Figure 3a). The fluorescence signal from the FCHA/PBS-treated skin was weak, indicating that the permeability of skin to FCHA itself is poor and it is unable to enter the SC layer. In contrast, strong fluorescence was observed for the skin treated with the FCHA-loaded reverse micelle formulation, and FCHA was distributed not only in the SC layer but also in the epidermis and the dermis layers. Consistent with these observations, the quantification of the FCHA extracted from the skin revealed that the reverse micelle formulation delivered 16.7 times more FCHA than the control aqueous solution (Figure 3b).
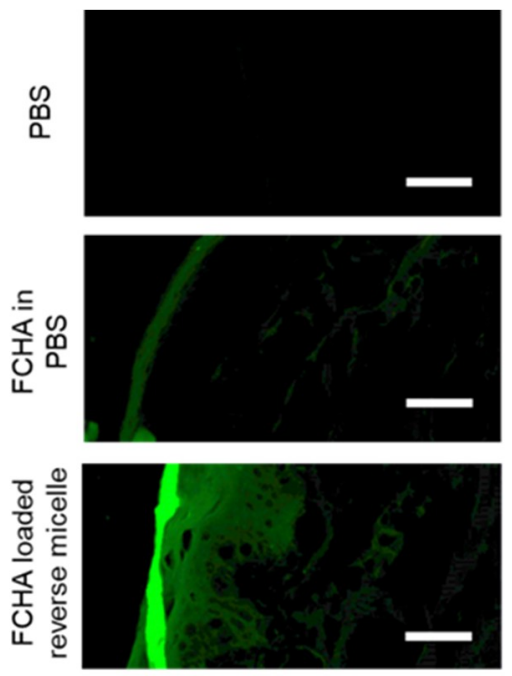

(a)

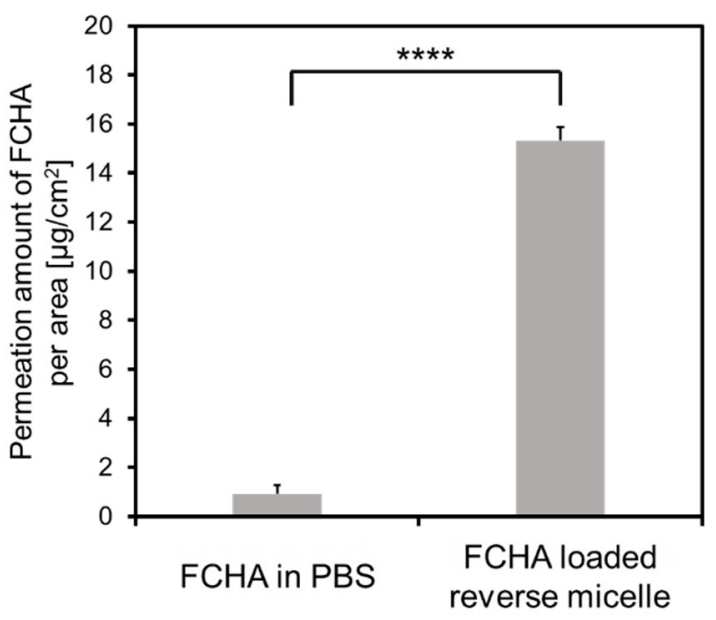

(b)

Figure 3. Skin permeability to FCHA. (a) CLSM images of the skin cross-sections of Yucatan micro pig (YMP) skin treated with FCHA/PBS or the FCHA-loaded reverse micelle formulation $((\mathrm{FCHA})=1.0 \mathrm{mg} / \mathrm{mL})$. The images were obtained with a $20 \times$ lens. Scale bars: $50 \mu \mathrm{m}$. (b) Amount of FCHA that permeated into the YMP skin, quantified from the fluorescence intensity of FCHA extracted from the skin. $\mathrm{n}=3$, mean $\pm \mathrm{SE},{ }^{* * * *} P<0.0001$.

\subsection{Permeation Mechanism of the FCHA-Loaded Reverse Micelle Formulation}

Further experiments were carried out to determine the underlying mechanism for the effective FCHA delivery by the reverse micelle formulation. The rate-limiting step of the mass transfer of the 
hydrophilic drug through the skin is the diffusion step within the hydrophobic SC layer; thus, we first observed how FCHA penetrates through the SC. In general, there are three possible pathways through the SC: the intracellular route, where a drug penetrates directly across corneocytes; the intercellular route, where a drug diffuses through the gap region between corneocytes; and the transappendageal route, where a drug diffuses via the hair follicles and sweat glands (Figure 4a) [28]. As can be seen in the CLSM images of the skin's top surface, green fluorescence highlighted the rims of the corneocytes, which clearly demonstrates that FCHA passed through the SC layer via the intercellular route (Figure 4b,c). Given that skin appendages occupy only $0.1 \%$ of the skin's surface, transappendageal penetration was not dominant in this case, although it is possible that it also contributed [29].

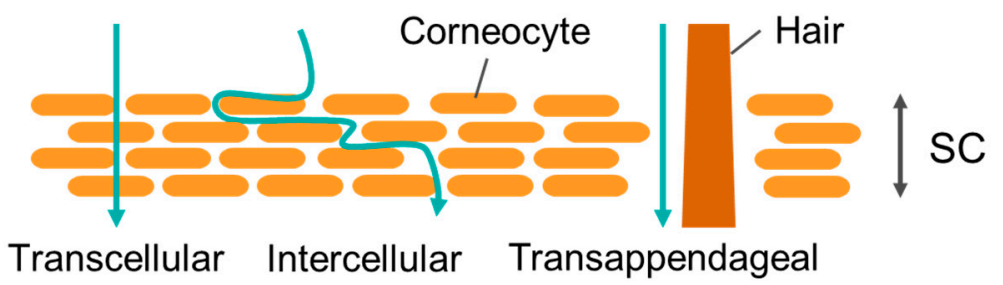

(a)

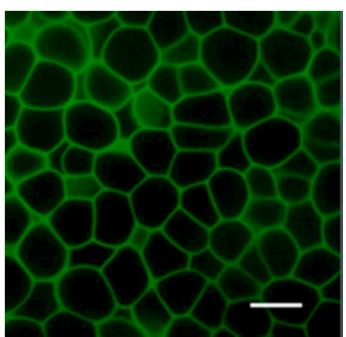

(b)

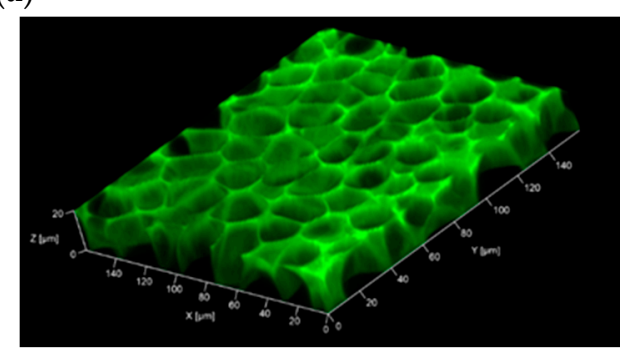

(c)

Figure 4. (a) Permeation pathways through the stratum corneum (SC) layer. (b) Top view of mouse ear skin treated with the FCHA-loaded reverse micelle formulation. The images were obtained with a $40 \times$ lens. Scale bar: $20 \mu \mathrm{m}$. (c) The 3D scanning of the SC layer.

FTIR spectroscopy was used to evaluate the effects of the FCHA-loaded reverse micelle formulation on the nanostructure of the intercellular region and establish why FCHA was able to penetrate through the SC layer [30]. The gap region surrounding the corneocytes is filled with ceramides, cholesterols and fatty acids that self-assemble to form lamellar structures. Information on the molecular orientation of these lipid molecules in the lamellar structure can be acquired from the peak positions of the $\mathrm{CH}_{2}$ symmetric stretching $\left(\sim 2850 \mathrm{~cm}^{-1}\right)$ and $\mathrm{CH}_{2}$ asymmetric stretching $\left(\sim 2920 \mathrm{~cm}^{-1}\right)$ in the FTIR spectrum. When the degree of molecular orientation decreases and the barrier function of the skin is weakened, the bent alkyl chain content increases, which manifests as blue shifts in the $\mathrm{CH}_{2}$ stretching modes [31,32]. We isolated the SC sheet from the YMP skin and treated it with FCHA-loaded reverse micelles for $24 \mathrm{~h}$. Figure 5 shows the FTIR spectra of pre- and post-treated SC sheets. Both $\mathrm{CH}_{2}$ stretching modes of the post-treated SC sheet were blue shifted by $2-3 \mathrm{~cm}^{-1}$, which indicates that the reverse micelle formulation altered the lamellar structure and enhanced the FCHA's diffusivity within the intercellular region. This is thought to be predominantly due to $\mathrm{MO}$, a skin permeation enhancer that has a kinked alkyl chain and is easily inserted into the lamellar structures $[33,34]$. HA also acts as a permeation enhancer; however, it only causes the hydration of the SC and does not alter the lipid nanostructure at low concentrations [11]. Judging from these data, FCHA was able to penetrate the SC layer via the intercellular route with the assistance of the reverse micelle formulation. 


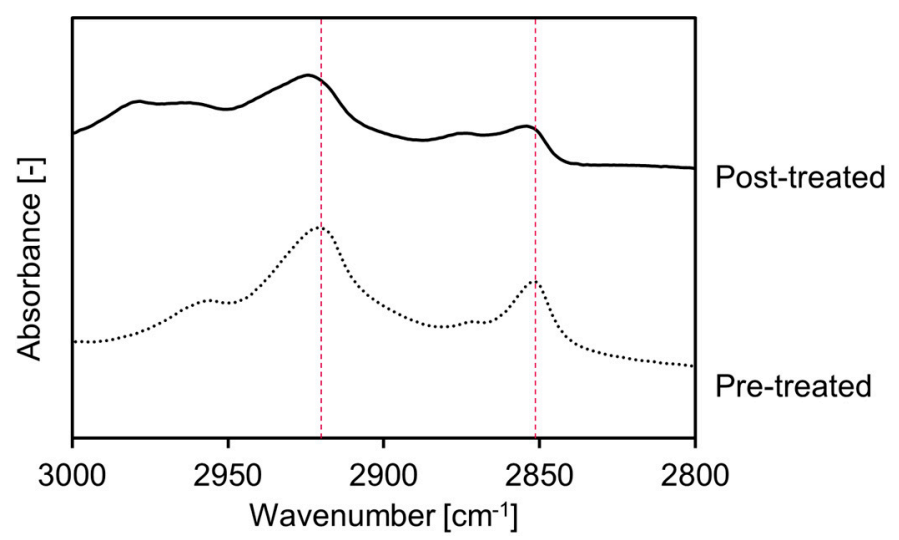

Figure 5. FTIR spectra of pre- and post-treated SC sheets. Peaks at around 2850 and $2920 \mathrm{~cm}^{-1}$ correspond to $\mathrm{CH}_{2}$ symmetrical and asymmetrical vibration modes of SC lipids in the intercellular region, respectively.

After passing through the SC, FCHA must permeate into the living epidermis layer. However, the FCHA encapsulated in the reverse micelles is not notionally expected to enter the hydrophilic part of the epidermis, owing to the hydrophobic nature of the reverse micelle surface. To reveal the mechanism in this step, we prepared dual-labeled reverse micelles using Rh-DOPE (0.015 wt.\%) as an indicator of MO (surfactant) and applied them to the YMP skin. Both green and red fluorescence were detected from the skin cross-section by CLSM observation (Figure 6a). While green fluorescence from FCHA was distributed to the deeper region, consistent with Figure 3, the red signal from Rh-DOPE was only detected from the top surface of the skin. The images support the notion of a mechanism of FCHA being released from the reverse micelles at the interface between the SC and the living epidermis, as illustrated in Figure $6 \mathrm{~b}$. In normal skin, the water content in the SC is $\sim 20 \%$, while that in the living epidermis is $~ 70 \%$ [35], which indicates that there is a pseudo-hydrophobic/hydrophilic interface between these layers. Due to the hydrophobic nature of MO (HLB = 3.8), the reverse micelles were unable to retain their morphology and collapsed before entering the living epidermis. Similar permeation behavior was also observed for another oil-based drug carrier [36].

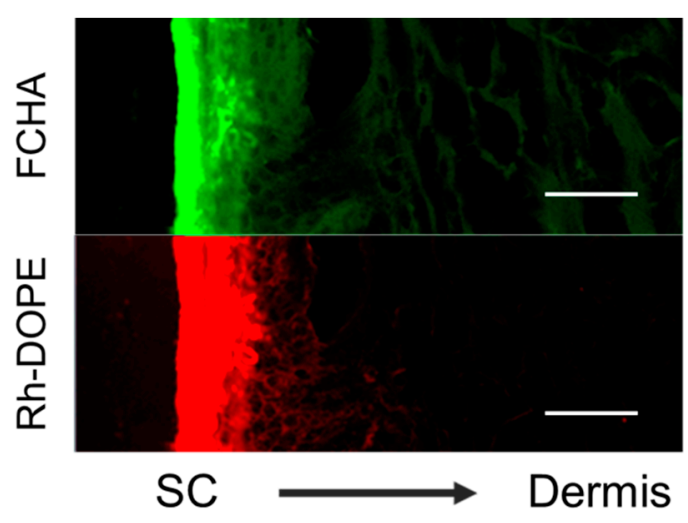

(a)

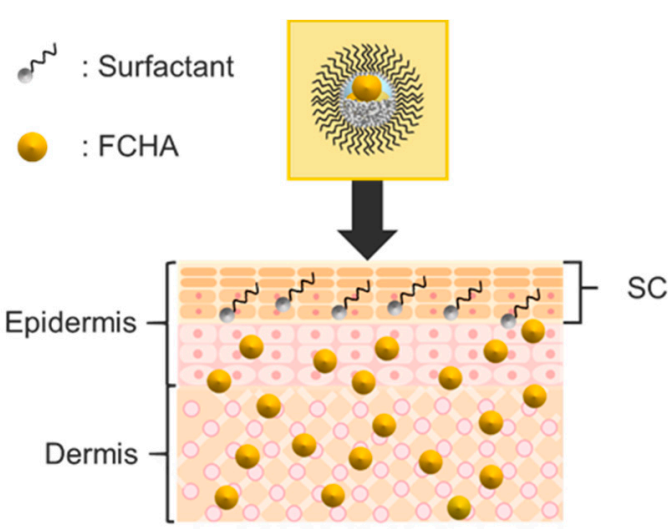

(b)

Figure 6. (a) CLSM images of the cross-section of YMP skin treated with a dual-labeled reverse micelle formulation (green: FCHA, red: Rh-DOPE). The images were obtained using a $20 \times$ lens. Scale bars: $50 \mu \mathrm{m}$. (b) Schematic illustration of the overall permeation mechanism of the FCHA-loaded reverse micelle formulation. 


\section{Conclusions}

The transcutaneous delivery of HA has been achieved using physical permeation enhancers and colloidal systems; however, these approaches have been limited by concerns for their safety, as well as their production costs. In this study, we used a reverse micelle formulation as an easy-to-prepare and effective transcutaneous delivery system for HA. The FCHA-loaded reverse micelle formulation was prepared by the simple mixing of IPM, IPA, MO and water, without any cumbersome processes such as freeze-drying or high-speed homogenizing. The mass fraction of IPA and the water-to-MO ratio influenced the homogeneity of the reverse micelles, and we successfully determined the optimal formulation in which the FCHA in the water phase was uniformly dispersed in IPM. The skin permeation of FCHA was significantly enhanced by the reverse micelle formulation and the fluorescent signal of FCHA was detected from the dermis layer of the YMP skin. Surface observation and FTIR spectroscopy of the skin revealed that the reverse micelle formulation disrupts the nanostructure of the intercellular lipids, allowing FCHA to penetrate the SC via the intercellular route. The permeation mechanism of FCHA was further investigated using a dual fluorescently-labeled reverse micelle formulation. The findings showed that FCHA was released from the reverse micelles at the hydrophobic/hydrophilic interface between the SC and the living epidermis. Our system was shown to be an easy-to-prepare formulation for the effective transcutaneous delivery of HA. The approach could be used in cosmetic and biomedical products and improve the quality of life for people with skin conditions.

Author Contributions: Conceptualization, T.N., T.U. and M.G.; methodology, A.K. and S.K.; investigation, A.K.; resources, R.W. and M.G.; data curation, A.K.; writing-original draft preparation, A.K. and S.K.; writing-review and editing, R.W. and M.G.; supervision, M.G.; project administration, M.G.; funding acquisition, R.W. and M.G. All authors have read and agreed to the published version of the manuscript.

Funding: Authors R.W. and M.G. received funding from a Grant-in-Aid for Scientific Research (S) 16H06369 from the Ministry of Education, Culture, Sports, Science, and Technology of Japan.

Acknowledgments: We thank Sarah Dodds, from Edanz Group (https://en-author-services.edanzgroup.com/) for editing a draft of this manuscript.

Conflicts of Interest: The authors declare no conflict of interest.

\section{References}

1. Meyer, L.J.; Sttern, R. Age-Dependent Changes of Hyaluronan in Human Skin. J. Invest. Dermatol. 1994, 102, 385-389. [CrossRef] [PubMed]

2. Takagi, Y.; Mori, K.; Taguchi, H.; Nishizaka, T.; Takema, Y. UVB sensitivity correlates with cutaneous barrier function in the skin of Japanese females. Photodermatol. Photoimmunol. Photomed. 2019, 35, $284-285$. [CrossRef] [PubMed]

3. Papakonstantinou, E.; Roth, M.; Karakiulakis, G. Hyaluronic acid: A key molecule in skin aging. Dermato-endocrinology 2012, 4, 253-258. [CrossRef]

4. Tang, M.; Svirskis, D.; Leung, E.; Kanamala, M.; Wang, H. Can intracellular drug delivery using hyaluronic acid functionalised $\mathrm{pH}$ - sensitive liposomes overcome gemcitabine resistance in pancreatic cancer? J. Control. Release 2019, 305, 89-100. [CrossRef] [PubMed]

5. Lee, Y.; Sugihara, K.; Gillilland, M.G.; Jon, S.; Kamada, N.; Moon, J.J. Hyaluronic acid-bilirubin nanomedicine for targeted modulation of dysregulated intestinal barrier, microbiome and immune responses in colitis. Nat. Mater. 2020, 19, 118-126. [CrossRef]

6. Cadete, A.; Olivera, A.; Besev, M.; Dhal, P.K.; Gonçalves, L.; Almeida, A.J.; Bastiat, G.; Benoit, J.P.; Garcia-Fuentes, M. Self-assembled hyaluronan nanocapsules for the intracellular delivery of anticancer drugs. Sci. Rep. 2019, 9, 1-11. [CrossRef]

7. He, M.-S.; Sheu, M.-M.; Huang, Z.-L.; Tsai, C.-H.; Tsai, R.-K. Sudden bilateral vision loss and brain infarction following cosmetic hyaluronic acid injection. JAMA Ophthalmol. 2013, 131, 1234-1235. [CrossRef]

8. Carle, M.V.; Roe, R.; Novack, R.; Boyer, D.S. Cosmetic Facial Fillers and Severe Vision Loss. JAMA Ophthalmol. 2014, 132, 637-639. [CrossRef] 
9. Bos, J.D.; Meinardi, M.M.H.M. The 500 Dalton rule for the skin penetration of chemical compounds and drugs. Exp. Dermatol. 2000, 9, 165-169. [CrossRef]

10. Zhu, J.; Tang, X.; Jia, Y.; Ho, C.; Huang, Q. Applications and delivery mechanisms of hyaluronic acid used for topical/transdermal delivery-A review. Int. J. Pharm. 2020, 578, 119127. [CrossRef]

11. Witting, M.; Boreham, A.; Brodwolf, R.; Alexiev, U.; Friess, W.; Hedtrich, S. Interactions of Hyaluronic Acid with the Skin and Implications for the Dermal Delivery of Biomacromolecules. Mol. Pharm. 2015, 12, 1391-1401. [CrossRef] [PubMed]

12. Vistoli, G.; Gennari, C.G.M.; Selmin, F.; Gardoni, F.; Franze, S.; Campisic, M.; Minghetti, P. The Role of the Conformational Profile of Polysaccharides on Skin Penetration: The Case of Hyaluronan and Its Sulfates. Chem. Biodivers. 2014, 11, 551-561.

13. Bok, M.; Zhao, Z.; Jeon, S.; Jeong, J.; Lim, E. Ultrasonically and Iontophoretically Enhanced Drug-Delivery System Based on Dissolving Microneedle Patches. Sci. Rep. 2020, 1-10. [CrossRef] [PubMed]

14. Kasetvatin, C.; Rujivipat, S.; Tiyaboonchai, W. Combination of elastic liposomes and low frequency ultrasound for skin permeation enhancement of hyaluronic acid. Colloids Surfaces B Biointerfaces 2015, 135, 458-464. [CrossRef]

15. Tokudome, Y.; Komi, T.; Omata, A.; Sekita, M. A new strategy for the passive skin delivery of nanoparticulate, high molecular weight hyaluronic acid prepared by a polyion complex method. Sci. Rep. 2018, 1-9. [CrossRef]

16. Funatsu, A.; Tahara, Y.; Yamanaka, S.; Goto, M. Oil Gel Sheets Utilizing Solid-in-Oil Technique. MEMBRANE 2011, 36, 57-62. [CrossRef]

17. Hoppel, M.; Juric, S.; Ettl, H.; Valenta, C. Effect of monoacyl phosphatidylcholine content on the formation of microemulsions and the dermal delivery of flufenamic acid. Int. J. Pharm. 2015, 479, 70-76. [CrossRef]

18. Lehtinen, O.; Wahyu, R.; Nugroho, N.; Lehtimaa, T.; Vierros, S.; Hiekkataipale, P.; Ruokolainen, J.; Sammalkorpi, M.; Österberg, M. Effect of temperature, water content and free fatty acid on reverse micelle formation of phospholipids in vegetable oil. Colloids Surfaces B Biointerfaces 2017, 160, 355-363. [CrossRef]

19. Kosakowska, K.A.; Casey, B.K.; Kurtz, S.L.; Lawson, L.B.; Grayson, S.M. Evaluation of Amphiphilic Star/Linear-Dendritic Polymer Reverse Micelles for Transdermal Drug Delivery: Directing Carrier Properties by Tailoring Core versus Peripheral Branching. Biomacromolecules 2018, 19, 3163-3176. [CrossRef]

20. Szuma, P.; Jungnickel, C.; Koz, K.; Jacyna, B.; Cal, K. Transdermal transport of collagen and hyaluronic acid using water in oil microemulsion. Int. J. Pharm. 2019, 572. [CrossRef]

21. Himes, R.; Lee, S.; Mcmenigall, K.; Russell-jones, G. The influence of molecular adjuvants in the cutaneous response to antigen after topical vaccination. Vaccine 2011, 29, 5393-5398. [CrossRef] [PubMed]

22. Kozaka, S.; Tahara, Y.; Wakabayashi, R.; Nakata, T.; Ueda, T.; Kamiya, N.; Goto, M. Transcutaneous Cancer Vaccine Using a Reverse Micellar Antigen Carrier. Mol. Pharm. 2020, 17, 645-655. [CrossRef] [PubMed]

23. Zakrewsky, M.; Lovejoy, K.S.; Kern, T.L.; Miller, T.E.; Le, V.; Nagy, A.; Goumas, A.M.; Iyer, R.S.; Del Sesto, R.E.; Koppisch, A.T.; et al. Ionic liquids as a class of materials for transdermal delivery and pathogen neutralization. Proc. Natl. Acad. Sci. USA 2014, 111, 13313-13318. [CrossRef] [PubMed]

24. Banerjee, A.; Ibsen, K.; Iwao, Y.; Zakrewsky, M.; Mitragotri, S. Transdermal Protein Delivery Using Choline and Geranate (CAGE) Deep Eutectic Solvent. Adv. Healthc. Mater. 2017, 6, 1-11. [CrossRef] [PubMed]

25. Kulkarni, C.V.; Wachter, W.; Iglesias-Salto, G.; Engelskirchen, S.; Ahualli, S. Monoolein: A magic lipid? Phys. Chem. Chem. Phys. 2011, 13, 3004-3021. [CrossRef]

26. Karande, P.; Jain, A.; Ergun, K.; Kispersky, V.; Mitragotri, S. Design principles of chemical penetration enhancers for transdermal drug delivery. Proc. Natl. Acad. Sci. USA 2005, 102, 4688-4693. [CrossRef]

27. Fujii, M.; Yamanouchi, S.; Hori, N.; Iwanaga, N.; Nawaguchi, N.; Matsumoto, M. Evaluation of Yucatan Micropig Skin for Use as an in Vitro Model for Skin Permeation Study. Biol. Pharm. Bull. 1997, 20, $249-254$. [CrossRef]

28. Kotla, N.G.; Chandrasekar, B.; Rooney, P.; Sivaraman, G.; Larrañaga, A.; Krishna, K.V.; Pandit, A.; Rochev, Y. Biomimetic Lipid-Based Nanosystems for Enhanced Dermal Delivery of Drugs and Bioactive Agents. ACS Biomater. Sci. Eng. 2017, 3, 1262-1272. [CrossRef]

29. Barry, B.W. Novel mechanisms and devices to enable successful transdermal drug delivery. Eur. J. Pharm. Sci. 2001, 14, 101-114. [CrossRef] 
30. Ruela, A.L.M.; Perissinato, A.G.; Lino, M.E.d.; Mudrik, P.S. Gislaine ribeiro pereira evaluation of skin absorption of drugs from topical and transdermal formulations. Braz. J. Pharm. Sci. 2014, 52, 527-544. [CrossRef]

31. Mendelsohn, R.; Flach, C.R.; Moore, D.J. Determination of molecular conformation and permeation in skin via IR spectroscopy, microscopy, and imaging. Biochim. Biophys. Acta-Biomembr. 2006, 1758, 923-933. [CrossRef] [PubMed]

32. Pouliot, R.; Germain, L.; Auger, A.; Tremblay, N. Julianna Juhasz Physical characterization of the stratum corneum of an in vitro human skin equivalent produced by tissue engineering and its comparison with normal human skin by ATR-FTIR spectroscopy and thermal analysis (DSC). Biochim. Biophys. Acta 1999, 1439, 341-352. [CrossRef]

33. Akinshina, A.; Das, C.; Noro, M.G. Effect of monoglycerides and fatty acids on a ceramide bilayer. Phys. Chem. Chem. Phys. 2016, 18, 17446-17460. [CrossRef]

34. Gupta, R.; Dwadasi, B.S.; Rai, B.; Mitragotri, S. Effect of Chemical Permeation Enhancers on Skin Permeability: In silico screening using Molecular Dynamics simulations. Sci. Rep. 2019, 9, 1456. [CrossRef]

35. Botelho, M.A.; Guerreiro, S.J.; Queiroz, D.B.; Barros, G.; Cavalcante, M.; Souza, J.M.O.; Silva, A.M.; Lemos, T.L.G.; Quintans, L., Jr. Depth-scanning confocal Raman for rapid in vivo determination of testosterone concentration profiles in human skin. Medicalexpress 2014, 1, 31-35. [CrossRef]

36. Tahara, Y.; Honda, S.; Kamiya, N.; Piao, H.; Hirata, A.; Hayakawa, E.; Fujii, T.; Goto, M. A solid-in-oil nanodispersion for transcutaneous protein delivery. J. Control. Release 2008, 131, 14-18. [CrossRef]

(C) 2020 by the authors. Licensee MDPI, Basel, Switzerland. This article is an open access article distributed under the terms and conditions of the Creative Commons Attribution (CC BY) license (http://creativecommons.org/licenses/by/4.0/). 